\title{
Finite-Time Synchronization and Identification between Two Nonlinear Coupled Multiweighted Markovian Switching Delayed Networks with Uncertain Models and External Disturbances
}

\author{
Xiaofei Chen, ${ }^{1}$ Haifeng Qiu $\mathbb{D D}^{2}{ }^{2}$ Chong $X u,{ }^{3}$ and Zhipeng Sun ${ }^{3}$ \\ ${ }^{1}$ College of Electrical Engineering, Zhejiang University, Hangzhou 310027, China \\ ${ }^{2}$ State Grid Hangzhou Xiaoshan Power Supply Company, Hangzhou 311200, China \\ ${ }^{3}$ State Grid Zhejiang Electric Power Company, Ltd., Hangzhou 310007, China
}

Correspondence should be addressed to Haifeng Qiu; 13857177475@sina.cn

Received 13 October 2020; Revised 24 November 2020; Accepted 1 December 2020; Published 17 December 2020

Academic Editor: Sanbo Ding

Copyright ( 2020 Xiaofei Chen et al. This is an open access article distributed under the Creative Commons Attribution License, which permits unrestricted use, distribution, and reproduction in any medium, provided the original work is properly cited.

In this paper, two different nonlinear coupled Markovian switching delayed network models with multiple weights are established, and the effects of parameter and model uncertainties and external disturbances are fully considered. Firstly, based on the finitetime theory, several sufficient conditions for the finite-time synchronization of Markovian switching networks are obtained. Secondly, under the feedback controller with adaptive laws, when the synchronization of the networks is achieved in a finite time, the uncertain parameters of the networks can also be identified as true values. Finally, a representative numerical simulation is given to further illustrate the validity and practicability of the theoretical analysis and proof of this paper.

\section{Introduction}

The complex dynamic network is a complex system with certain states and functions that is composed of many nodes and the topological connections formed by the edges between the nodes. In addition, as an important class of nonlinear systems, complex networks have received extensive attention from all walks of life. Researchers studying complex systems in the form of complex networks can not only deepen their understanding of the structure of complex systems but also gain a deeper understanding of complex systems in nature and society (including power grid, Internet, transportation network, and communication network) $[1,2]$. In the research of complex networks, synchronization of complex networks has always been one of the hot topics. This is mainly because the synchronization phenomenon has a vital impact on real life. For example, the synchronization of the power grid ensures the safe and reliable operation of the power system, and the synchronization of the transportation network ensures the safety and smoothness of the road. Generally speaking, synchronization can be divided into inner synchronization and outer synchronization. In order to achieve global robust exponential synchronization and global robust asymptotical synchronization of drive response-based discontinuous Cohen-Grossberg neural networks, the pinning state-feedback and adaptive controllers were designed in [3]. In [4], a boundary controller relying to distributed measurement was designed to achieve nonlinear time-delayed complex spatiotemporal network (CSN). Compared with the inner synchronization, there are relatively few studies on out synchronization. Outer synchronization means that two or more systems with completely the same or similar properties gradually approach and reach the same state through the interaction of the systems under different initial conditions. Therefore, this paper mainly studies the outer synchronization between two different complex networks.

It is well known that the network topology has an important influence on the network synchronization. This is because different topological structures have different dynamic states. At present, the research on fixed topology has 
made great achievements. However, the network topology is always affected by various factors in practice, such as the influence of network attacks, electromagnetic interference, and equipment damage, and the network topology will randomly change under the influence of these factors $[5,6]$. This kind of random switching can depend on the Markovian process, so the research on the synchronization of Markovian switching networks is more practical [7-9]. In [10], the authors studied the finite-time synchronization of the Markovian jump complex dynamical framework with hybrid coupling. Cluster synchronization of Markovian switching complex networks with hybrid couplings and stochastic perturbations was studied in [11]. In addition, the Markovian switching complex networks considered above are all modeled with a single weight. But many real networks are complex systems with multiple weights, and each node of these systems is connected through multiple weights $[12,13]$. Therefore, in order to make the network models closer to the real system, the Markovian switching network models with multiple weights should be considered.

In the real world, the coupling relationship of Markovian switching networks cannot be all linear, and nonlinear coupling systems are more common. In some cases, the variable $x(t)$ cannot be directly obtained, but only $h(x(t))$ can be obtained, and the synchronization of the system can only be realized by $h(x(t))$ at this time. Therefore, the synchronization of nonlinear coupled systems also has great research value, and the results are more practical. In [14], the authors concerned with the problem of synchronization in nonlinear complex networks with multiple time-varying delays via adaptive aperiodic intermittent control. In [4], the authors dealt with the problem for synchronization of a nonlinear time-delayed complex spatiotemporal network (CSN), modeled by semilinear parabolic partial differentialdifference equations. Furthermore, in the actual signal transmission process, time delays are often caused by factors, such as transmission speed limitations, network information congestion, and the influence of the transmission environment $[15,16]$. Thus, considering the above reasons, time delays should be considered in the synchronization of Markovian switching complex networks.

Most of the system models considered above are deterministic. It is well known that the parameters of the real system may be difficult to determine in advance, and the parameters of the system are generally unknown or uncertain [17]. In addition, under the influence of certain external environments and system structure changes, there may be some uncertain models and external disturbances in the dynamics of the system. The synchronization process of the system may be affected or even destroyed by these uncertainties. The robust finite-time state estimation problem of the uncertain Markovian jump neural networks with partly unknown transition probabilities was investigated in [18]. In [19], the effects of parameter and model uncertainties and external disturbances were fully taken into account, and a sliding model control approach was introduced to realize modified projective synchronization between two different chaotic systems in a finite time. Therefore, it is necessary to study the synchronization between Markovian switching complex networks with uncertainty.

In actual engineering, people not only pay attention to its gradual stability characteristics in infinite time but also are more interested in realizing the synchronization state of the system in a finite time, such as aerospace systems, robot control systems, and power systems; they are often required that the synchronization be reached in a finite time. The finite-time control technology can realize this idea, and the finite-time control scheme can make the network have better robustness [20]. In [15], the author investigated finite-time synchronization of complexed-valued neural networks with multiple time-varying delays and infinite distributed delays. The multiweighted coupled neural network (MWCNN) models with and without coupling delays were investigated in [21]. Therefore, it is also valuable to realize the finite-time synchronization of Markovian switching complex networks in practice.

Based on the above discussion, this paper mainly studied the finite-time synchronization and identification problems of nonlinear coupled Markovian switching delayed networks with multiple weights, in presence of uncertain parameters, uncertain model, and external disturbances on the networks. To the best of our knowledge, there are rarely studies on the finite-time synchronization and parameters identification between two nonlinear coupled Markovian switching delayed networks with multiple weights and uncertainties. Therefore, the research has significance in terms of theory and practice.

The rest of this paper is arranged as follows. In Section 2, some notations and necessary conditions will be given. The theoretical proof of network finite-time synchronization and identification is given in Section 3. In Section 4, in order to prove the validity of the above theoretical analysis, a representative numerical example is given. The conclusions are drawn in Section 5.

\section{Preliminaries}

In order to complete the theoretical proof and analysis of this paper, some important mathematical symbols and necessary conditions are given in this section. First of all, some important mathematical symbol definitions will be given. The matrix $A^{T}$ (or $x^{T}$ ) is defined as the transposed matrix of $A$ (or $x$ ). The symbol $\otimes$ is defined as the Kronecker product of matrixes. Define $\|x\|_{2}$ as the 2-norm of $x$. Define $I_{n} \in R^{n \times n}$ as the $n$-dimensional identity matrix. $E$ is defined as mathematical expectation. Secondly, some necessary conditions will be given below.

Assumption 1. Assume that a Lipschitz condition is satisfied by noise intensity function $\sigma_{i}\left(t, e_{i}(t), r(t)\right)$, and there exists a constant $\rho_{i} \geq 0$ such that 


$$
\operatorname{trace}\left[\sigma_{i}^{T}\left(t, e_{i}(t), r(t)\right) \sigma_{i}\left(t, e_{i}(t), r(t)\right)\right] \leq \rho_{i}(r(t)) e_{i}^{T}(t) e_{i}(t)
$$

Assumption 2. For arbitrary $x(t), y(t) \in R^{n}$, if there exists a constant $L>0$, then following inequality holds:

$$
\|f(y(t))-f(x(t))\| \leq L\|(y(t)-x(t))\| .
$$

Assumption 3. (see [22]). Let $0<\gamma<1$ and $\kappa>0$; assume that there exists a continuous function $g(g(0)>0)$ such that, for any $0 \leq u \leq t$,

$$
g(t)-g(u) \leq-\kappa \int_{u}^{t}(g(s))^{\gamma} \mathrm{d} s .
$$

Lemma 1 (see [23]). Assume that a positive definite, continuous function $V(t)$ satisfies the differential inequality $\dot{V}(t) \leq-\alpha V^{\varepsilon}(t), \forall t \geq t_{0}, V\left(t_{0}\right) \geq 0$, where $\alpha>0,0<\varepsilon<1$. For any given $t_{0}, V(t)$ satisfies the inequality $V^{1-\varepsilon}(t) \leq V^{1-\varepsilon}\left(t_{0}\right)-\alpha(1-\varepsilon)\left(t-t_{0}\right), t_{0} \leq t \leq t_{1}$, and $V(t)$ $\equiv 0, \forall t \geq t_{1}$; we can get $t_{1}$ :

$$
t_{1}=t_{0}+\frac{V^{1-\varepsilon}\left(t_{0}\right)}{\alpha(1-\varepsilon)} .
$$

Lemma 2 (see [23]). For any vectors $x(t), y(t) \in R^{n}$ and $a$ positive definite matrix $K>0$, the following inequality holds:

$$
2 x^{T} y \leq x^{T} K^{-1} x+y^{T} K y .
$$

Lemma 3 (see [24]). $b_{1}, b_{2}, \ldots, b_{n} \in R_{n}$ are any vectors, and the following inequality holds:

$$
\left|b_{1}\right|^{c}+\left|b_{2}\right|^{c}+\cdots+\left|b_{n}\right|^{c} \geq\left(\left|b_{1}\right|^{2}+\left|b_{2}\right|^{2}+\cdots+\left|b_{n}\right|^{2}\right)^{c / 2},
$$

i.e.,

$$
\sum_{i=1}^{n}\left|b_{i}\right|^{c} \geq\left(\sum_{i=1}^{n}\left|b_{i}\right|^{2}\right)^{c / 2}
$$

where $c(0<c<2)$ is a real number.

Definition 1 (see [24]). Define $f: R^{n} \times R^{+} \times S \longrightarrow R^{n}$ and $g: R^{n} \times R_{+} \times S \longrightarrow R^{n \times m}$ are differentiable functions satisfying the following $n$-dimensional functional stochastic differential equation:

$$
\mathrm{d} x(t)=f(x(t), t, r(t)) \mathrm{d} t+g(x(t), t,(r(t))) \mathrm{d} \omega(t) .
$$

According to Itô's formula, if $V(x(t), t, r)$ $\in C^{2,1}\left(R^{n} \times R_{+} \times S \longrightarrow R_{+}\right)$, the operator $\mathscr{L} V$ is defined as

$$
\begin{aligned}
\mathscr{L} V(x(t), t, p)= & V_{t}(x(t), t, p)+V_{x}(x(t), t, p) f(x(t), t, p) \\
& +\frac{1}{2} \operatorname{trace}\left[g^{T}(x(t), t, p) V_{x x} g(x(t), t, p)\right]+\sum_{q=1}^{m} \pi_{p q} V(x(t), t, q),
\end{aligned}
$$

where $V_{t}(x(t), t, p)=(\partial V(x(t), t, p)) / \partial t, \quad V_{x}(x(t), t, p)$ $=\left(\left((\partial(x(t), t, p)) / \partial x_{1}\right), \ldots,\left((\partial(x(t), t, p)) / \partial x_{n}\right)\right)$, and $V_{x x}(x(t), t, p)=\left(\left(\partial^{2} V(x(t), t, p)\right) / \partial x_{i} x_{j}\right)_{n \times n}$.

\section{Main Results}

In this section, the nonlinear coupled Markovian switching delayed network models with multiple weights and uncertainties will be established, and the feedback controller with update laws is designed; then, the finite-time synchronization and identification of the network models is achieved by above controller and some inequality techniques.

3.1. Network Models. In this paper, the $n$-dimensional drive and response networks with uncertain parameters, models, and external disturbances are given as follows:

The drive network is as follows:

$$
\left.\dot{x}_{i}(t)=f_{i 1}\left(x_{i}(t), x_{i}(t-\tau)\right)\right)+f_{i 2}\left(x_{i}(t), x_{i}(t-\tau)\right) \alpha_{i}(t)+\sum_{d=1}^{l} \sum_{j=1}^{N} a_{i j}^{d}(r(t)) \Gamma_{d} h\left(x_{j}(t)\right)+\Delta f_{i 1}\left(x_{i}(t)+\Delta_{i 1}, \quad i=1,2, \ldots, N\right.
$$

where $x_{i}(t)=\left(x_{i 1}(t), x_{i 2}(t), \ldots, x_{i n}(t)\right)^{T} \in R^{n}$ is the state vector of the $i$-th node; $f_{i 1}: R^{n} \longrightarrow R^{n}$ and $f_{i 2}: R^{n} \longrightarrow R^{n \times n}$ are continuous vector function and matrix function, respectively; $\Delta f_{i 1}: R^{n} \longrightarrow R^{n}$ is the model uncertainty; $h(\cdot): R^{n} \longrightarrow R^{n}$ is the nonlinear coupling function; $\alpha_{i}(t)$ is an unknown parameter vector of the dynamic node; $\tau>0$ is the time delays of node $i ; \Delta_{i 1}$ is external disturbances of the network; and $\Gamma_{d} \in R^{n \times n}(d=1,2, \ldots, l)$ is the inner coupling matrix of the $d$-th coupling form. $A^{d}(r(t))=\left(a_{i j}^{d}(r(t))\right)_{N \times N} \in R^{N \times N}$ is the topological structure matrix of the $d$-th coupling form, which represents topological structure of the network at time $t$ in mode $r(t)$, 
and $A^{d}(r(t))$ can be defined as follows: if there exists a connection between node $i$ and node $j(j \neq i)$ for the $d$-th coupling form, then $a_{i j}^{d} \neq 0$; otherwise, $a_{i j}^{d}=0$. The diagonal elements of $A(r(t))$ are defined as

$$
a_{i i}^{d}(r(t))=-\sum_{j=1, j \neq i}^{N} a_{i j}^{d}(r(t)), \quad d=1,2, \ldots, l ; i=1,2, \ldots, N .
$$

Let $\{r(t), t \geq 0\}$ be a right-continuous Markovian process in probability space, which takes values in a finite space $S=$ $\{1,2, \ldots, l\}$ with generator $\Pi=\left(\pi_{p q}\right)_{m \times m}$. The transition probability from the $p$-th mode at time $t$ to the $q$-th mode at time $t+\Delta t$ is defined as follows:

$$
P\{r(t+\Delta t)=q \mid r(t)=p\}= \begin{cases}\pi_{p q} \Delta t+o(\Delta t), & \text { if } q \neq p, \\ 1+\pi_{p p} \Delta t+o(\Delta t), & \text { if } q=p\end{cases}
$$

where $\Delta t>0, \lim _{\Delta t \longrightarrow 0} o(\Delta t) / \Delta t=0$, and transition rate $\pi_{p q}\left(\pi_{p q}>0\right)$ satisfies

$$
\pi_{p p}=-\sum_{q=1, q \neq p}^{m} \pi_{p q} .
$$

$$
\begin{aligned}
\dot{y}_{i}(t)= & g_{i 1}\left(y_{i}(t), y_{i}(t-\tau)\right)+g_{i 2}\left(y_{i}(t), y_{i}(t-\tau)\right) \beta_{i}(t)+\sum_{d=1}^{l} \sum_{j=1}^{N} b_{i j}^{d}(r(t)) \Gamma_{d} h\left(y_{j}(t)\right)+\Delta_{i 2}+\Delta g_{i 1}\left(y_{i}(t)\right) \\
& +\sigma_{i}\left(t, e_{i}(t), r(t)\right) \dot{\omega}(t)+u_{i}(t), \quad i=1,2, \ldots, N
\end{aligned}
$$

The response network is as follows:$$
\lim _{t \longrightarrow t_{1}} E\left\|e_{i}(t)\right\|=\lim _{t \longrightarrow t_{1}} E\left\|y_{i}(t)-x_{i}(t)\right\|_{2}=0, \quad i=1,2, \ldots, N
$$

where $y_{i}(t)=\left(y_{i 1}(t), y_{i 2}(t), \ldots, y_{\text {in }}(t)\right)^{T} \in R^{n}$ is the state vector of the $i$-th node; $g_{i 1}: R^{n} \longrightarrow R^{n}$ and $g_{i 2}: R^{n} \longrightarrow R^{n \times n}$ are continuous vector function and matrix function, respectively; $\Delta g_{i 1}: R^{n} \longrightarrow R^{n}$ is the model uncertainty; $\beta_{i}(t)$ is an unknown parameter vector of the dynamic node with the same definition as $\alpha_{i}(t) ; \Delta_{i 2}$ is the external disturbances of the network; $B^{d}(r(t))=\left(b_{i j}^{d}(r(t))\right)_{N \times N} \in R^{N \times N}$ is the same as the definition of $A^{d}(r(t))$ above; and $B^{d}(r(t))$ can be defined as follows: if there exists a connection between node $i$ and node $j(j \neq i)$ for the $d$-th coupling form, then $b_{i j}^{d} \neq 0$; otherwise, $b_{i j}^{d}=0$. The diagonal elements of $B(r(t))$ is defined as

$$
b_{i i}^{d}(r(t))=-\sum_{j=1, j \neq i}^{N} b_{i j}^{d}(r(t)), \quad d=1,2, \ldots, l ; i=1,2, \ldots, N .
$$

Moreover, $u_{i}(t)$ is a nonlinear controller; $\omega(t)=\left(\omega_{1}(t), \omega_{2}(t), \ldots, \omega_{n}(t)\right)^{T}$ is a $n$-dimensional Weiner process.

Define synchronization error of the system as follows:

$$
e_{i}(t)=y_{i}(t)-x_{i}(t), \quad i=1,2, \ldots, N,
$$

where $e_{i}(t)=\left(e_{i 1}(t), e_{i 2}(t), \ldots, e_{i n}(t)\right)^{T}$ represents the state error of the $i$-th node.

Definition 2. If there is a positive constant $t$ and gives an arbitrary $t_{1}>t$, such that and $\left\|e_{i}(t)\right\|_{2}=\left\|y_{i}(t)-x_{i}(t)\right\|_{2} \equiv 0$, then synchronization of Markovian switching complex networks (10) and (14) can be realized at a finite-time $t_{1}$.

Remark 1. When the drive system (10) and the response system (14) gradually realize synchronization at a finite-time $t_{1}$, the unknown parameter vectors $\alpha_{i}(t)$ and $\beta_{i}(t)$ will be identified and expressed as

$$
\left\{\begin{array}{l}
\lim _{t \longrightarrow t_{1}} \sum_{i=1}^{N}\left(\left\|\widehat{\alpha}_{i}(t)-\alpha_{i}\right\|_{2}\right)=0 \\
\lim _{t \longrightarrow t_{1}} \sum_{i=1}^{N}\left(\left\|\widehat{\beta}_{i}(t)-\beta_{i}\right\|_{2}\right)=0
\end{array}\right.
$$

where $\widehat{\alpha}_{i}(t)$ and $\widehat{\beta}_{i}(t)$ are estimated values of $\alpha_{i}(t)$ and $\beta_{i}(t)$, respectively.

3.2. Main Results. In this section, finite-time synchronization between two different nonlinear coupled Markovian switching complex delayed networks with multiple weights and uncertainties will be demonstrated and analyzed.

According to network equations (10) and (14), the synchronization error equation of system can be obtained: 


$$
\begin{aligned}
\dot{e}_{i}(t)= & g_{i 1}\left(y_{i}(t), y_{i}(t-\tau)\right)+\Delta g_{i 1}\left(y_{i}(t)\right)+g_{i 2}\left(y_{i}(t), y_{i}(t-\tau)\right) \beta_{i}(t)+\Delta_{i 2}-\Delta_{i 1} \\
& -f_{i 1}\left(x_{i}(t), x_{i}(t-\tau)\right)-f_{i 2}\left(x_{i}(t), x_{i}(t-\tau)\right) \alpha_{i}(t)+\sigma_{i}\left(t, e_{i}(t), r(t)\right) \dot{\omega}(t) \\
& -\Delta f_{i 1}\left(x_{i}(t)\right)+\sum_{d=1}^{l} \sum_{j=1}^{N} b_{i j}^{d}(r(t)) \Gamma_{d} h\left(y_{j}(t)\right)-\sum_{d=1}^{l} \sum_{j=1}^{N} a_{i j}^{d}(r(t)) \Gamma_{d} h\left(x_{j}(t)\right)+u_{i}(t) .
\end{aligned}
$$

Obviously, when the synchronization error (19) converges to zero in a finite time, then finite-time synchronization and identification of the network models will also be achieved. Therefore, it is very important to design an effective controller so that the synchronization error converges to zero.

Theorem 1. Design an effective controller with update laws as follows:

$$
\left\{\begin{array}{l}
u_{i}(t)=-\eta_{i}(r(t)) e_{i}(t)-g_{i 1}\left(y_{i}(t), y_{i}(t-\tau)\right)-g_{i 2}\left(y_{i}(t), y_{i}(t-\tau)\right) \widehat{\beta}_{i}(t) \\
+f_{i 1}\left(x_{i}(t), x_{i}(t-\tau)\right)+f_{i 2}\left(x_{i}(t), x_{i}(t-\tau)\right) \widehat{\alpha}_{i}(t)-k(r(t)) \operatorname{sign}\left(e_{i}(t)\right)\left|e_{i}(t)\right|^{\gamma} \\
+\sum_{d=1}^{l} \sum_{j=1}^{N}\left(a_{i j}^{d}(r(t))-b_{i j}^{d}(r(t))\right) \Gamma_{d} h\left(x_{j}(t)\right)-\widehat{\Delta}_{i} \operatorname{sign}\left(e_{i}(t)\right)-\widehat{v}_{i} \operatorname{sign}\left(e_{i}(t)\right), \\
\dot{\widehat{\alpha}}_{i}(t)=-\lambda_{i}(r(t)) f_{i 2}^{T}\left(x_{i}(t), x_{i}(t-\tau)\right) e_{i}(t)-\psi_{i}(r(t)) \operatorname{sign}\left(\widehat{\alpha}_{i}(t)\right)\left(\left|\widehat{\alpha}_{i}(t)\right|+M_{\alpha}\right)^{\gamma}, \\
\dot{\hat{\beta}}_{i}(t)=\mu_{i}(r(t)) g_{i 2}^{T}\left(y_{i}(t), y_{i}(t-\tau)\right) e_{i}(t)-\psi_{i}(r(t)) \operatorname{sign}\left(\widehat{\beta}_{i}(t)\right)\left(\left|\widehat{\beta}_{i}(t)\right|+M_{\beta}\right)^{\gamma}, \\
\dot{\widehat{\Delta}}_{i}(t)=\varphi_{i}(r(t)) e_{i}^{T}(t) \operatorname{sign}\left(e_{i}(t)\right)-\psi_{i}(r(t)) \operatorname{sign}\left(\widehat{\Delta}_{i}(t)\right)\left(\left|\widehat{\Delta}_{i}(t)\right|+M_{\triangle}\right)^{\gamma} \\
\hat{\widehat{v}}_{i}(t)=\phi_{i}(r(t)) e_{i}^{T}(t) \operatorname{sign}\left(e_{i}(t)\right)-\psi_{i}(r(t)) \operatorname{sign}\left(\widehat{v}_{i}(t)\right)\left(\left|\widehat{v}_{i}(t)\right|+M_{v}\right)^{\gamma},
\end{array}\right.
$$

where control parameters $\eta_{i}(r(t))>0, \quad k_{i}(r(t))>0$, $\lambda_{i}(r(t))>0, \quad \mu_{i}(r(t))>0, \quad \varphi_{i}(r(t))>0, \quad \phi_{i}(r(t))>0, \quad$ and $\psi_{i}(r(t))>0,(r \in S) ; M_{\alpha}, M_{\beta}, M_{\Delta}$, and $M_{v}$ are, respectively, known constants satisfying conditions $M_{\alpha} \geq\left|\alpha_{i}\right|, M_{\beta} \geq\left|\beta_{i}\right|$, $M_{\Delta} \geq\left|\Delta_{i}\right|$, and $M_{v} \geq\left|v_{i}\right|$, if the following inequality holds:

$$
\left\{\begin{array}{l}
\sum_{q=1}^{m} \pi_{p q}(D(q)-Q(p)) \leq 0, \\
\widetilde{K}+\Omega+\left(\frac{1}{2} \Theta(r(t))+\widetilde{O}-\Xi(r(t))\right) \otimes I_{m} \leq 0,
\end{array}\right.
$$

where $D(q)$ is a positive definite matrix of appropriate dimension, $Q(q)$ is an arbitrary symmetric matrix, $\Theta(r(t))=$ $\operatorname{diag}\left\{\rho_{1}(r(t)), \rho_{2}(r(t)), \ldots, \rho_{N}(r(t))\right\} ; \operatorname{sign}\left(e_{i}(t)\right)=\operatorname{diag}$ $\left\{\operatorname{sign}\left(e_{i 1}(t)\right), \operatorname{sign}\left(e_{i 2}(t)\right), \ldots, \operatorname{sign}\left(e_{i n}(t)\right)\right\} ; \Omega=(1 / 2) \sum_{d=1}^{l}$ $\left(B^{d} \otimes \Gamma_{d}\right) K_{d}^{-1}\left(B^{d} \otimes \Gamma_{d}\right)^{T}, \omega_{p}=\sum_{q=1}^{m}(D(q)-Q(p)) / 2, \quad \widetilde{\mathrm{O}}=$
$(1 / 2) l L_{1}^{2}\left\|K_{d}\right\| I_{N} ; \widetilde{\mathrm{K}}=I_{N} \otimes \varpi_{p}$, and $\Xi(r(t))=\operatorname{diag}\left\{\eta_{1}(r(t))\right.$, $\left.\eta_{2}(r(t)), \ldots, \eta_{\mathrm{N}}(r(t))\right\}$.

Then, finite-time synchronization between two different nonlinear coupled multiweighted Markovian switching 
delayed networks (10) and (14) can be achieved under the controller (20) and inequality condition (21). According to Lemma 1 , the finite-time $t_{1}$ can be expressed as follows:

$$
t_{1} \leq t_{0}+\frac{V\left(t_{0}, r\left(t_{0}\right)\right)^{1-\varepsilon}}{\xi 2^{\varepsilon}(1-\varepsilon)},
$$

where $V\left(t_{0}, r\left(t_{0}\right)\right)$ is the function value of $V(t, r(t))$ at the initial time and $\xi=\min \left\{\min (k), \min (\psi), \kappa / 2^{(1+\gamma) / 2}\right\}$.

Proof. Construct the Lyapunov function as follows:

$$
\begin{aligned}
V(t, e(t), p)= & \frac{1}{2} \sum_{i=1}^{N} e_{i}^{T}(t) e_{i}(t)+\frac{1}{2} \sum_{i=1}^{N} \frac{1}{\lambda_{i}(p)} \widetilde{\alpha}_{i}^{T}(t) \widetilde{\alpha}_{i}(t)+\frac{1}{2} \sum_{i=1}^{N} \frac{1}{\mu_{i}(p)} \widetilde{\beta}_{i}^{T}(t) \tilde{\beta}_{i}(t) \\
& \cdot \frac{1}{2} \sum_{i=1}^{N} \frac{1}{\varphi_{i}(p)} \widetilde{\Delta}_{i}^{2}(t)+\frac{1}{2} \sum_{i=1}^{N} \frac{1}{\phi_{i}(p)} \widetilde{v}_{i}^{2}(t)+\sum_{i=1}^{N} \int_{t-\tau}^{t} e_{i}^{T}(\theta) e_{i}(\theta) \mathrm{d} \theta
\end{aligned}
$$

where $\widetilde{\alpha}_{i}(t), \widetilde{\beta}_{i}(t), \widetilde{v}_{i}(t)$, and $\widetilde{\Delta}_{i}(t)$ are the error between the true value and the estimated value.
According to Definition 1, the differential operator $\mathscr{L}$ can be given as

$$
\begin{aligned}
\mathscr{L} V(t, e(t), p)= & \sum_{i=1}^{N} e_{i}^{T}(t) \dot{e}_{i}(t)+\sum_{i=1}^{N} \frac{1}{\lambda_{i}(p)} \dot{\widetilde{\alpha}}_{i}^{T}(t) \widetilde{\alpha}_{i}(t)+\sum_{i=1}^{N} \frac{1}{\mu_{i}(p)} \dot{\tilde{\beta}}_{i}^{T}(t) \widetilde{\beta}_{i}(t) \\
& +\sum_{i=1}^{N} \frac{1}{\varphi_{i}(p)} \widetilde{\Delta}_{i}(t) \dot{\widetilde{\Delta}}_{i}(t)+\sum_{i=1}^{N} \frac{1}{\phi_{i}(p)} \widetilde{v}_{i}(t) \dot{\widetilde{v}}_{i}(t)+\sum_{i=1}^{N} e_{i}^{T}(t) e_{i}(t) \\
& -\sum_{i=1}^{N} e_{i}^{T}(t-\tau) e_{i}(t-\tau)+\sum_{q=1}^{m} \pi_{p q} \sum_{i=1}^{N} \int_{t-\tau}^{t} e_{i}^{T}(\theta) e_{i}(\theta) \mathrm{d} \theta \\
& +\frac{1}{2} \sum_{i=1}^{N} \operatorname{trace}\left[\sigma_{i}^{T}\left(t, e_{i}(t), p\right) \sigma_{i}\left(t, e_{i}(t), p\right)\right]+\sum_{q=1}^{m} \pi_{p q} \frac{1}{2} \sum_{i=1}^{N} e_{i}^{T}(t) e_{i}(t) .
\end{aligned}
$$

Taking the controller (20) into (24), we can get

$$
\begin{aligned}
\mathscr{L} V(t, e(t), p)= & \sum_{i=1}^{N} e_{i}^{T}(t)\left\{-g_{i 2}\left(y_{i}(t), y_{i}(t-\tau)\right) \widetilde{\beta}_{i}(t)+f_{i 2}\left(x_{i}(t), x_{i}(t-\tau)\right) \widetilde{\alpha}_{i}(t)-\eta_{i}(p) e_{i}(t)\right. \\
& +\sum_{d=1}^{l} \sum_{j=1}^{N} b_{i j}^{d}(p) \Gamma_{d}\left(h\left(y_{j}(t)\right)-h\left(x_{j}(t)\right)\right)-\widehat{\Delta}_{i} \operatorname{sign}\left(e_{i}(t)\right)-\widehat{v}_{i} \operatorname{sign}\left(e_{i}(t)\right)+\Delta_{i 2} \\
& \left.-\Delta_{i 1}-k(p) \operatorname{sign}\left(e_{i}(t)\right)\left|e_{i}(t)\right|^{\gamma}+\Delta g_{i 1}\left(y_{i}(t)\right)-\Delta f_{i 1}\left(x_{i}(t)\right)\right\}+\sum_{i=1}^{N} e_{i}^{T}(t) e_{i}(t) \\
& +\sum_{q=1}^{m} \pi_{p q} \frac{1}{2} \sum_{i=1}^{N} e_{i}^{T}(t) D(q) e_{i}(t)+\frac{1}{2} \sum_{i=1}^{N} \operatorname{trace}\left[\sigma_{i}^{T}\left(t, e_{i}(t), p\right) \sigma_{i}\left(t, e_{i}(t), p\right)\right] \\
& -\sum_{i=1}^{N} e_{i}^{T}(t) f_{i 2}\left(x_{i}(t), x_{i}(t-\tau)\right) \widetilde{\alpha}_{i}(t)+\sum_{i=1}^{N} e_{i}^{T}(t) g_{i 2}\left(y_{i}(t), y_{i}(t-\tau)\right) \tilde{\beta}_{i}(t) \\
& -\psi(p) \sum_{i=1}^{N} \frac{1}{\lambda_{i}(p)} \operatorname{sign}\left(\widehat{\alpha}_{i}(t)\right)\left(\left|\widehat{\alpha}_{i}(t)\right|+M_{\alpha}\right)^{\gamma} \widetilde{\alpha}_{i}(t)+\sum_{i=1}^{N} \widetilde{\Delta}_{i}(t) e_{i}^{T}(t) \operatorname{sign}\left(e_{i}(t)\right)
\end{aligned}
$$




$$
\begin{aligned}
& -\psi(p) \sum_{i=1}^{N} \frac{1}{\mu_{i}(p)} \operatorname{sign}\left(\widehat{\beta}_{i}(t)\right)\left(\left|\widehat{\beta}_{i}(t)\right|+M_{\beta}\right)^{\gamma} \widetilde{\beta}_{i}(t)+\sum_{i=1}^{N} \widetilde{v}_{i}(t) e_{i}^{T}(t) \operatorname{sign}\left(e_{i}(t)\right) \\
& -\psi(p) \sum_{i=1}^{N} \frac{1}{\varphi_{i}(p)} \operatorname{sign}\left(\widehat{\Delta}_{i}(t)\right)\left(\left|\widehat{\Delta}_{i}(t)\right|+M_{\Delta}\right)^{\gamma} \widetilde{\Delta}_{i}(t)-\sum_{i=1}^{N} e_{i}^{T}(t-\tau) e_{i}(t-\tau) \\
& -\psi(p) \sum_{i=1}^{N} \frac{1}{\phi_{i}(p)} \operatorname{sign}\left(\widehat{v}_{i}(t)\right)\left(\left|\widehat{v}_{i}(t)\right|+M_{v}\right)^{\gamma} \widetilde{v}_{i}(t) \\
& +\sum_{q=1}^{m} \pi_{p q} \sum_{i=1}^{N} \int_{t-\tau}^{t} e_{i}^{T}(\theta) D(q) e_{i}(\theta) \mathrm{d} \theta .
\end{aligned}
$$

In addition, if uncertainties $\Delta f_{1}\left(x_{i}(t)\right), \Delta g_{1}\left(y_{i}(t)\right)$ and disturbances $\Delta_{i 1}$ and $\Delta_{i 2}$ are all bounded, then there exist non-negative constants $v$ and $\Delta$, then

$$
\begin{array}{r}
\left\|\Delta g_{1}\left(y_{i}(t)\right)-\Delta f_{1}\left(x_{i}(t)\right)\right\| \leq v \leq M_{v} \\
\left\|\Delta_{i 2}-\Delta_{i 1}\right\| \leq \Delta \leq M_{\Delta} .
\end{array}
$$

By Assumption 1 and (26), equation (25) can be simplified as follows:

$$
\begin{aligned}
\mathscr{L} V(t, e(t), p)= & \sum_{i=1}^{N} e_{i}^{T}(t)\left\{-\eta_{i}(p) e_{i}(t)-k(p) \operatorname{sign}\left(e_{i}(t)\right)\left|e_{i}(t)\right|^{\gamma}+\sum_{d=1}^{l} \sum_{j=1}^{N} b_{i j}^{d}(p) \Gamma_{d} H\left(e_{j}(t)\right)\right\} \\
& +\sum_{q=1}^{m} \pi_{p q} \sum_{i=1}^{N} \int_{t-\tau}^{t} e_{i}^{T}(\theta) D(q) e_{i}(\theta) \mathrm{d} \theta+\sum_{q=1}^{m} \pi_{p q} \frac{1}{2} \sum_{i=1}^{N} e_{i}^{T}(t) D(q) e_{i}(t) \\
& -\psi(p) \sum_{i=1}^{N} \frac{1}{\lambda_{i}(p)} \operatorname{sign}\left(\widehat{\alpha}_{i}(t)\right)\left(\left|\widehat{\alpha}_{i}(t)\right|+M_{\alpha}\right)^{\gamma} \widetilde{\alpha}_{i}(t)-\sum_{i=1}^{N} e_{i}^{T}(t-\tau) e_{i}(t-\tau) \\
& -\psi(p) \sum_{i=1}^{N} \frac{1}{\mu_{i}(p)} \operatorname{sign}\left(\widehat{\beta}_{i}(t)\right)\left(\left|\widehat{\beta}_{i}(t)\right|+M_{\beta}\right)^{\gamma} \widetilde{\beta}_{i}(t)+\sum_{i=1}^{N} e_{i}^{T}(t) e_{i}(t) \\
& -\psi(p) \sum_{i=1}^{N} \frac{1}{\phi_{i}(p)} \operatorname{sign}\left(\widehat{v}_{i}(t)\right)\left(\left|\widehat{v}_{i}(t)\right|+M_{v}\right)^{\gamma} \widetilde{v}_{i}(t)+\frac{1}{2} \rho_{i}(r(t)) e_{i}^{T}(t) e_{i}(t) \\
& -\psi(p) \sum_{i=1}^{N} \frac{1}{\varphi_{i}(p)} \operatorname{sign}\left(\widehat{\Delta}_{i}(t)\right)\left(\left|\widehat{\Delta}_{i}(t)\right|+M_{\Delta}\right)^{\gamma} \widetilde{\Delta}_{i}(t),
\end{aligned}
$$

where $H\left(e_{j}(t)\right)=h\left(y_{j}(t)\right)-h\left(x_{j}(t)\right)$.

According to Lemma 2 and Assumption 2, we can get

$$
\begin{aligned}
\sum_{i=1}^{N} e_{i}^{T}(t) \sum_{d=1}^{l} \sum_{j=1}^{N} b_{i j}^{d}(p) \Gamma_{d} H\left(e_{j}(t)\right) & =\sum_{d=1}^{l} e^{T}(t)\left(B^{d} \otimes \Gamma_{d}\right) H(e(t)) \\
& \leq \frac{1}{2} \sum_{d=1}^{l} e^{T}(t)\left(B^{d} \otimes \Gamma_{d}\right) K_{d}^{-1}\left(B^{d} \otimes \Gamma_{d}\right)^{T} e(t)+\frac{1}{2} l H^{T}(e(t))\left\|K_{d}\right\| H(e(t)) \\
& \leq \frac{1}{2} \sum_{d=1}^{l} c_{d} e^{T}(t)\left(B^{d} \otimes \Gamma_{d}\right) K_{d}^{-1}\left(B^{d} \otimes \Gamma_{d}\right)^{T} e(t)+\frac{1}{2} l L_{1}^{2}\left\|K_{d}\right\| e^{T}(t) e(t) .
\end{aligned}
$$


According to Assumption 3, and bringing the inequality (28) into inequality (27), we can get

$$
\begin{aligned}
\mathscr{L} V(t, e(t), p) \leq & \frac{1}{2} l L_{1}^{2}\left\|K_{d}\right\| \sum_{i=1}^{N} e_{i}^{T}(t) e_{i}(t)-k(p) \sum_{i=1}^{N} e_{i}^{T}(t) \operatorname{sign}\left(e_{i}(t)\right)\left|e_{i}(t)\right|^{\gamma} \\
& -\kappa \sum_{i=1}^{N} \int_{t-\tau}^{t}\left(e_{i}^{T}(\theta) e_{i}(\theta)\right) \frac{1+\gamma}{2} \mathrm{~d} \theta+\sum_{q=1}^{m} \pi_{p q} \frac{1}{2} \sum_{i=1}^{N} e_{i}^{T}(t) D(q) e_{i}(t) \\
& -\psi(p) \sum_{i=1}^{N} \frac{1}{\lambda_{i}(p)} \operatorname{sign}\left(\widehat{\alpha}_{i}(t)\right)\left(\left|\widehat{\alpha}_{i}(t)\right|+M_{\alpha}\right)^{\gamma} \widetilde{\alpha}_{i}(t) \\
& +\frac{1}{2} \rho_{i}(r(t)) e_{i}^{T}(t) e_{i}(t)+\sum_{q=1}^{m} \pi_{p q} \sum_{i=1}^{N} \int_{t-\tau}^{t} e_{i}^{T}(\theta) D(q) e_{i}(\theta) \mathrm{d} \theta \\
& -\psi(p) \sum_{i=1}^{N} \frac{1}{\mu_{i}(p)} \operatorname{sign}\left(\widehat{\beta}_{i}(t)\right)\left(\left|\widehat{\beta}_{i}(t)\right|+M_{\beta}\right)^{\gamma} \widetilde{\beta}_{i}(t) \\
& -\psi(p) \sum_{i=1}^{N} \frac{1}{\phi_{i}(p)} \operatorname{sign}\left(\widehat{v}_{i}(t)\right)\left(\left|\widehat{v}_{i}(t)\right|+M_{v}\right)^{\gamma} \widetilde{v}_{i}(t)-\eta_{i}(p) \sum_{i=1}^{N} e_{i}^{T}(t) e_{i}(t) \\
& -\psi(p) \sum_{i=1}^{N} \frac{1}{\varphi_{i}(p)} \operatorname{sign}\left(\widehat{\Delta}_{i}(t)\right)\left(\left|\widehat{\Delta}_{i}(t)\right|+M_{\Delta}\right)^{\gamma} \widetilde{\Delta}_{i}(t) .
\end{aligned}
$$

Based on Lemma 3, we have

$$
\begin{aligned}
-\sum_{i=1}^{N} e_{i}^{T}(t) \operatorname{sign}\left(e_{i}(t)\right)\left|e_{i}(t)\right|^{\gamma} & =-\sum_{i=1}^{N} \sum_{j=1}^{N} e_{i j}(t)\left|e_{i j}(t)\right|^{\gamma} \operatorname{sign}\left(e_{i j}(t)\right)=-\sum_{i=1}^{N} \sum_{j=1}^{N}\left|e_{i j}(t)\right|^{1+\gamma} \\
& \leq-\left(\sum_{i=1}^{N} \sum_{j=1}^{N}\left|e_{i j}(t)\right|^{2}\right)^{1+\gamma / 2}=-\left(\sum_{i=1}^{N} e_{i}^{T}(t) e_{i}(t)\right)^{1+\gamma / 2}
\end{aligned}
$$

From some inequality relationships, we can obtain Hence,

$\left|\widetilde{\alpha}_{i}(t)\right|=\left|\widehat{\alpha}_{i}(t)-\alpha_{i}(t)\right| \leq\left|\widehat{\alpha}_{i}(t)\right|+\left|\alpha_{i}(t)\right| \leq\left|\widehat{\alpha}_{i}(t)\right|+M_{\alpha}$.

$$
\begin{aligned}
& -\sum_{i=1}^{N}\left(\left|\widehat{\alpha}_{i}(t)\right|+M_{\alpha}\right)^{\gamma} \operatorname{sign}\left(\widehat{\alpha}_{i}(t)\right) \widetilde{\alpha}_{i}(t)=-\sum_{i=1}^{N}\left(\left|\widehat{\alpha}_{i}(t)\right|+M_{\alpha}\right)^{\gamma} \widetilde{\alpha}_{i}(t) \\
\leq & -\sum_{i=1}^{N}\left|\widehat{\alpha}_{i}(t)-\alpha_{i}(t)\right|^{\gamma} \widetilde{\alpha}_{i}(t)=-\sum_{i=1}^{N}\left|\widetilde{\alpha}_{i}(t)\right|^{\gamma+1} \leq-\left(\sum_{i=1}^{N} \widetilde{\alpha}_{i}^{T}(t) \widetilde{\alpha}_{i}(t)\right)^{(1+\gamma / 2)}, \\
& -\sum_{i=1}^{N}\left(\left|\widehat{\beta}_{i}(t)\right|+M_{\beta}\right)^{\gamma} \operatorname{sign}\left(\widehat{\beta}_{i}(t)\right) \widetilde{\beta}_{i}(t)=-\sum_{i=1}^{N}\left(\left|\widehat{\beta}_{i}(t)\right|+M_{\beta}\right)^{\gamma} \widetilde{\beta}_{i}(t) \\
\leq & -\sum_{i=1}^{N}\left|\widehat{\beta}_{i}(t)-\beta_{i}(t)\right|^{\gamma} \widetilde{\beta}_{i}(t)=-\sum_{i=1}^{N}\left|\widetilde{\beta}_{i}(t)\right|^{\gamma+1} \leq-\left(\sum_{i=1}^{N} \widetilde{\beta}_{i}^{T}(t) \widetilde{\beta}_{i}(t)\right)^{(1+\gamma / 2)},
\end{aligned}
$$




$$
\begin{aligned}
& -\sum_{i=1}^{N}\left(\left|\widehat{\Delta}_{i}(t)\right|+M_{\Delta}\right)^{\gamma} \operatorname{sign}\left(\widehat{\Delta}_{i}(t)\right) \widetilde{\Delta}_{i}(t)=-\sum_{i=1}^{N}\left(\left|\widehat{\Delta}_{i}(t)\right|+M_{\Delta}\right)^{\gamma} \widetilde{\Delta}_{i}(t) \\
\leq & -\sum_{i=1}^{N}\left|\widehat{\Delta}_{i}(t)-\Delta_{i}(t)\right|^{\gamma} \widetilde{\Delta}_{i}(t)=-\sum_{i=1}^{N}\left|\widetilde{\Delta}_{i}(t)\right|^{\gamma+1} \leq-\left(\sum_{i=1}^{N} \widetilde{\Delta}_{i}^{2}(t)\right)^{(1+\gamma / 2)}, \\
- & \sum_{i=1}^{N}\left(\left|\widehat{v}_{i}(t)\right|+M_{v}\right)^{\gamma} \operatorname{sign}\left(\widehat{v}_{i}(t)\right) \widetilde{v}_{i}(t)=-\sum_{i=1}^{N}\left(\left|\widehat{v}_{i}(t)\right|+M_{v}\right)^{\gamma} \widetilde{v}_{i}(t) \\
\leq & -\sum_{i=1}^{N}\left|\widehat{v}_{i}(t)-v_{i}(t)\right|^{\gamma} \widetilde{v}_{i}(t)=-\sum_{i=1}^{N}\left|\widetilde{v}_{i}(t)\right|^{\gamma+1} \leq-\left(\sum_{i=1}^{N} \widetilde{v}_{i}^{2}(t)\right)^{\frac{1+\gamma}{2}} .
\end{aligned}
$$

According to inequalities (30) and (32)-(35), we can get

$$
\begin{aligned}
\mathscr{L} V(t, e(t), p) \leq & -k(p)\left(\sum_{i=1}^{N} e_{i}^{T}(t) e_{i}(t)\right)^{(1+\gamma / 2)}-\kappa\left(\sum_{i=1}^{N} \int_{t-\tau}^{t}\left(e_{i}^{T}(\theta) e_{i}(\theta)\right)\right)^{(1+\gamma / 2)} \\
& -\psi(p)\left(\sum_{i=1}^{N} \frac{1}{\lambda_{i}(p)} \widetilde{\alpha}_{i}^{T}(t) \widetilde{\alpha}_{i}(t)\right)^{(1+\gamma / 2)}-\psi(p)\left(\sum_{i=1}^{N} \frac{1}{\mu_{i}(p)} \widetilde{\beta}_{i}^{T}(t) \widetilde{\beta}_{i}(t)\right)^{(1+\gamma / 2)} \\
& -\psi(p)\left(\sum_{i=1}^{N} \frac{1}{\varphi_{i}(p)} \widetilde{\Delta}_{i}^{2}(t)\right)^{(1+\gamma / 2)}-\psi(p)\left(\sum_{i=1}^{N} \frac{1}{\phi_{i}(p)} \widetilde{v}_{i}^{2}(t)\right)^{(1+\gamma / 2)}-\eta_{i}(p) \sum_{i=1}^{N} e_{i}^{T}(t) e_{i}(t) \\
& +\frac{1}{2} l L_{1}^{2}\left\|K_{d}\right\| \sum_{i=1}^{N} e_{i}^{T}(t) e_{i}(t)+\sum_{q=1}^{m} \pi_{p q} \frac{1}{2} \sum_{i=1}^{N} e_{i}^{T}(t)(D(q)-Q(p)) e_{i}(t) \\
& +\frac{1}{2} \rho_{i}(r(t)) e_{i}^{T}(t) e_{i}(t)+\sum_{q=1}^{m} \pi_{p q} \sum_{i=1}^{N} \int_{t-\tau}^{t} e_{i}^{T}(\theta)(D(q)-Q(p)) e_{i}(\theta) \mathrm{d} \theta .
\end{aligned}
$$

From Lemma 3 and inequality (21), we can get

$$
\begin{aligned}
\mathscr{L} V(t, e(t), p) \leq & \sum_{i=1}^{N} e_{i}^{T}(t)\left\{\widetilde{\mathrm{K}}+\Omega+\left(\frac{1}{2} \Theta(r(t))+\widetilde{\mathrm{O}}-\Xi(r(t))\right) \otimes I_{n}\right\} e_{i}(t) \\
& -\xi\left(\sum_{i=1}^{N} e_{i}^{T}(t) e_{i}(t)+\sum_{i=1}^{N} \frac{1}{\lambda_{i}(p)} \widetilde{\alpha}_{i}^{T}(t) \widetilde{\alpha}_{i}(t)+\sum_{i=1}^{N} \frac{1}{\mu_{i}(p)} \widetilde{\beta}_{i}^{T}(t) \widetilde{\beta}_{i}(t)\right. \\
& \left.+\sum_{i=1}^{N} \frac{1}{\varphi_{i}(p)} \widetilde{\Delta}_{i}^{2}(t)+\sum_{i=1}^{N} \frac{1}{\phi_{i}(p)} \widetilde{v}_{i}^{2}(t)+2 \sum_{i=1}^{N} \int_{t-\tau}^{t} e_{i}^{T}(\theta) e_{i}(\theta) \mathrm{d} \theta\right)^{(1+\gamma / 2)} \\
= & \sum_{i=1}^{N} e_{i}^{T}(t) P e_{i}(t)-\xi 2 \frac{1+\gamma}{2} \frac{1+\gamma}{2}(t, e(t), p) .
\end{aligned}
$$

According to conditions (21), the above inequality (37) can be written as

$$
\mathscr{L} V(t, e(t), p) \leq-\xi 2^{\varepsilon} V^{\varepsilon}(t, e(t), p),
$$

where $\varepsilon=(1+\gamma / 2)$.
From Lemma 1 and inequality (22), if $t_{0}=0$, finite-time $t_{1}$ can be estimated as

$$
t_{1} \leq \frac{V(0, r(0))^{1-\varepsilon}}{\theta 2^{\varepsilon}(1-\varepsilon)} .
$$


Remark 2. Based on an effective controller with update laws, when networks (10) and (14) achieve synchronization in a finite-time $t_{1}$, the unknown parameter vectors $\alpha_{i}(t)$ and $\beta_{i}(t)$ of the networks will be identified as true value.

Remark 3. In the finite-time synchronization and parameters identification process between two nonlinear coupled multiweighted Markovian switching delayed networks with uncertainties (10) and (14), inequality (21) is only a sufficient condition, not a necessary condition.

Remark 4. In the synchronization and parameters identification process between two Markovian switching networks, the speed of synchronization and unknown parameters identification will be affected by these control parameters $\eta_{i}, k, \lambda_{i}, \mu_{i}, \varphi, \phi_{i}, \psi_{i}, \Delta_{i}, v_{i}$ in controller (20).

\section{Simulink Results}

In this section, a representative mathematical simulation is given to prove the validity and feasibility of the above theoretical analysis. Firstly, the node dynamics equations of the driving network can be described by a chaotic system:

$$
\left\{\begin{array}{l}
\dot{x}_{i 1}(t)=\alpha_{i 1}\left(x_{i 2}(t)-x_{i 1}(t)\right) \\
\dot{x}_{i 2}(t)=\alpha_{i 3} x_{i 1}(t)-x_{i 1}(t) x_{i 3}(t)-x_{i 2}(t), \\
\dot{x}_{i 3}(t)=x_{i 1}(t) x_{i 2}(t)-\beta_{i 2} x_{i 3}(t)
\end{array}\right.
$$

The Lorenz system is taken as the node dynamic of driving complex network, and system (40) can be expressed as

$$
\begin{aligned}
\left(\begin{array}{c}
\dot{x}_{i 1}(t) \\
\dot{x}_{i 2}(t) \\
\dot{x}_{i 3}(t)
\end{array}\right)= & \left(\begin{array}{c}
0 \\
-x_{i 1}(t) x_{i 3}(t)-x_{i 2}(t) \\
x_{i 1}(t) x_{i 2}(t)
\end{array}\right) \\
& +\left(\begin{array}{ccc}
x_{i 2}(t)-x_{i 1}(t) & 0 & 0 \\
0 & 0 & x_{i 2}(t) \\
0 & -x_{i 3}(t) & 0
\end{array}\right) \times\left(\begin{array}{c}
\alpha_{i 1} \\
\alpha_{i 2} \\
\alpha_{i 3}
\end{array}\right),
\end{aligned}
$$

where the identification value of the unknown parameter vector $\alpha_{i}$ is $\alpha_{i}=\left(\alpha_{i 1}, \alpha_{i 2}, \alpha_{i 3}\right)^{T}=(10,8 / 3,28)^{T}$.

Secondly, the node dynamics equations of the response network can be described by a chaotic system:

$$
\left\{\begin{array}{l}
\dot{y}_{i 1}(t)=\beta_{i 1}\left(y_{i 2}(t)-y_{i 1}(t)\right) \\
\dot{y}_{i 2}(t)=\left(\beta_{i 3}-\beta_{i 1}\right) y_{i 1}(t)-y_{i 1}(t) y_{i 3}(t)+\beta_{i 3} y_{i 2}(t), \\
\dot{y}_{i 3}(t)=y_{i 1}(t) y_{i 2}(t)-\beta_{i 2} y_{i 3}(t)
\end{array}\right.
$$

The Chen system is taken as the node dynamic of response complex network, and the system (42) can be expressed as

$$
\left(\begin{array}{c}
\dot{y}_{i 1}(t) \\
\dot{y}_{i 2}(t) \\
\dot{y}_{i 3}(t)
\end{array}\right)=\left(\begin{array}{c}
0 \\
-y_{i 1}(t) y_{i 3}(t) \\
y_{i 1}(t) y_{i 2}(t)
\end{array}\right)+\left(\begin{array}{ccc}
y_{i 2}(t)-y_{i 1}(t) & 0 & 0 \\
-y_{i 1}(t) & 0 & y_{i 1}(t)+y_{i 2}(t) \\
0 & -y_{i 3}(t) & 0
\end{array}\right) \times\left(\begin{array}{l}
\beta_{i 1} \\
\beta_{i 2} \\
\beta_{i 3}
\end{array}\right),
$$

where the identification value of the unknown parameter vector $\beta_{i}$ is $\beta_{i}=\left(\beta_{1}, \beta_{2}, \beta_{3}\right)^{T}=(35,3,28)^{T}$.

The number of system nodes is 20 in numerical simulation. Moreover, Figure 1 shows the system mode switching process. The other relevant parameters are as follows:

$$
\begin{aligned}
& l=2, h(x)=\sin (x), \Gamma_{1}=\left[\begin{array}{lll}
1 & 0 & 0 \\
0 & 1 & 0 \\
0 & 0 & 1
\end{array}\right], \Gamma_{2}=\left[\begin{array}{lll}
2 & 0 & 0 \\
0 & 2 & 0 \\
0 & 0 & 2
\end{array}\right], \\
& \Delta f_{1}\left(x_{i}(t)\right)=\quad\left(\begin{array}{l}
0.1 \cos \left(x_{1}(t)\right) \\
0.2 \cos \left(x_{2}(t)\right) \\
0.3 \cos \left(x_{3}(t)\right)
\end{array}\right), \quad \Delta g_{1}\left(x_{i}(t)\right)= \\
& \left(\begin{array}{l}
0.1 \sin \left(x_{1}(t)\right) \\
0.2 \sin \left(x_{2}(t)\right) \\
0.3 \sin \left(x_{3}(t)\right)
\end{array}\right), \quad \Delta_{1}=\left(\begin{array}{l}
\cos \left(0.1 x_{1}(t)\right) \\
\cos \left(0.2 x_{2}(t)\right) \\
\cos \left(0.3 x_{3}(t)\right)
\end{array}\right), \quad \text { and } \Delta_{2}= \\
& \left(\begin{array}{l}
\sin \left(0.1 x_{1}(t)\right) \\
\sin \left(0.2 x_{2}(t)\right) \\
\sin \left(0.3 x_{3}(t)\right)
\end{array}\right) .
\end{aligned}
$$

In the follow, according to the controller (20) and condition (21), some control parameters of controller (20) are selected as $\eta(1)=\eta(2)=10 ; k(1)=12, k(2)=15$; $\lambda(1)=\lambda(2)=15 ; \quad \mu(1)=24, \quad \mu(2)=18 ; \quad \varphi(1)=\varphi(2)=5$; $\psi(1)=9, \quad \psi(2)=4 ; \phi(1)=\phi(2)=15 ; \gamma=0.6 ; \quad M_{\alpha}=100$, $M_{\beta}=100, M_{\alpha}=100$, and $M_{v}=100$. In this simulation process, the start time of the simulation is selected at $t_{0}=0$ and time delay $\tau=0.01$. In addition, through Lemma 1 and (39), we can obtain synchronization time $t_{1} \leq 9.3287$.

The time-varying curves of networks synchronization errors $e_{i}(t)(i=1,2,3)$ are shown in Figure 2. In Figure 2, synchronization error curves of networks gradually converge to 0 . Moreover, Figure 3 is the identification process of the unknown parameter vectors $\alpha_{i}(i=1,2,3)$, and the unknown parameter vector is finally identified as $\alpha_{i}=(10,8 / 3,28)^{T}$. Figure 4 is the identification process of the unknown parameter vectors $\beta_{i}(i=1,2,3)$, and the unknown parameter vector is finally identified as $\beta_{i}=(35,3,28)^{T}$. From the curve change trend in Figures $2-4$, it can be seen that the networks synchronization and parameter identification are achieved at about $t=7$. By comparing calculated values with simulated values, the theoretical proof of the third part is also verified. 


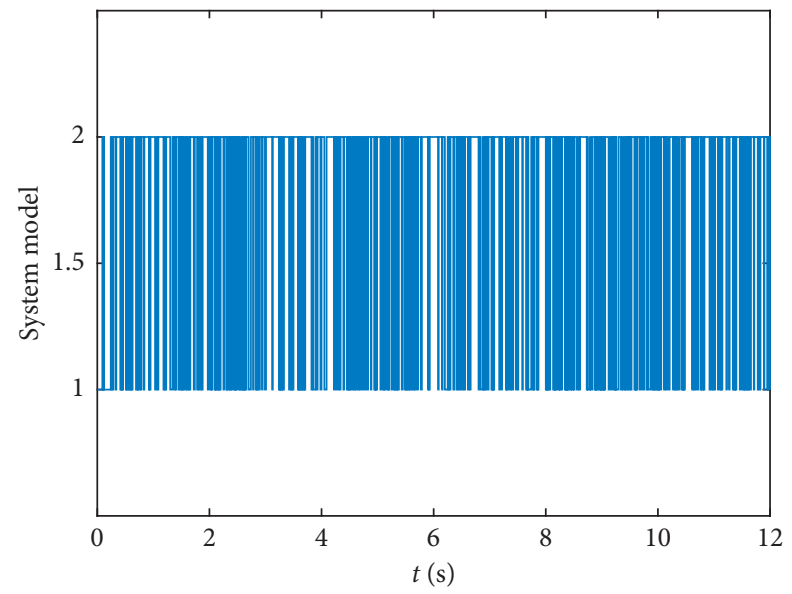

FIGURE 1: Switching of system mode.

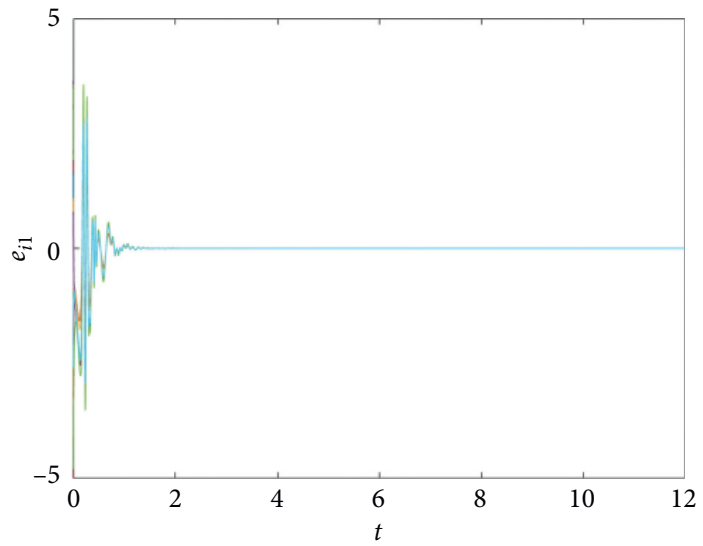

(a)

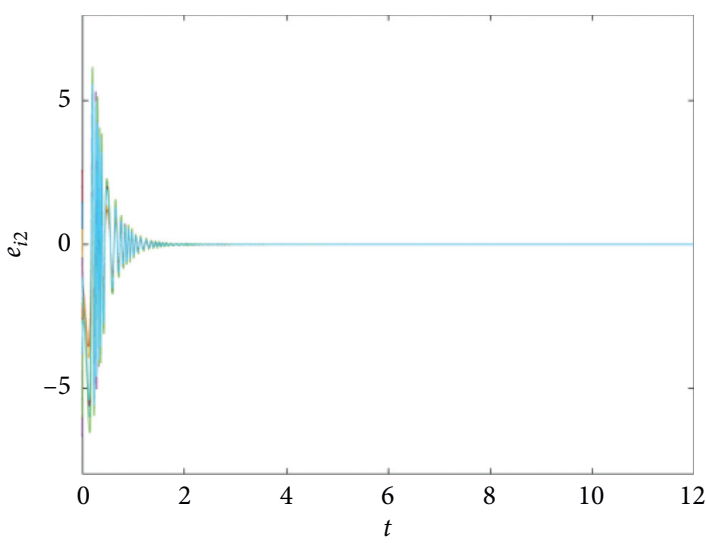

(b)

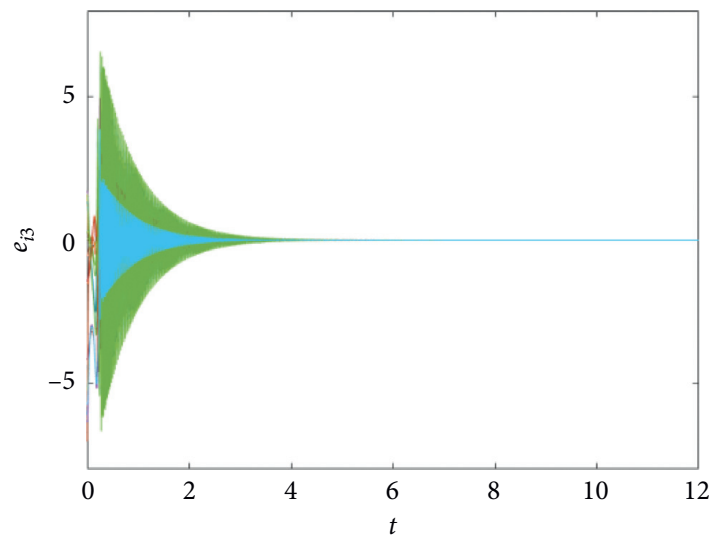

(c)

FIgURE 2: Synchronization errors $e_{i}(t)$ with system evolution. 


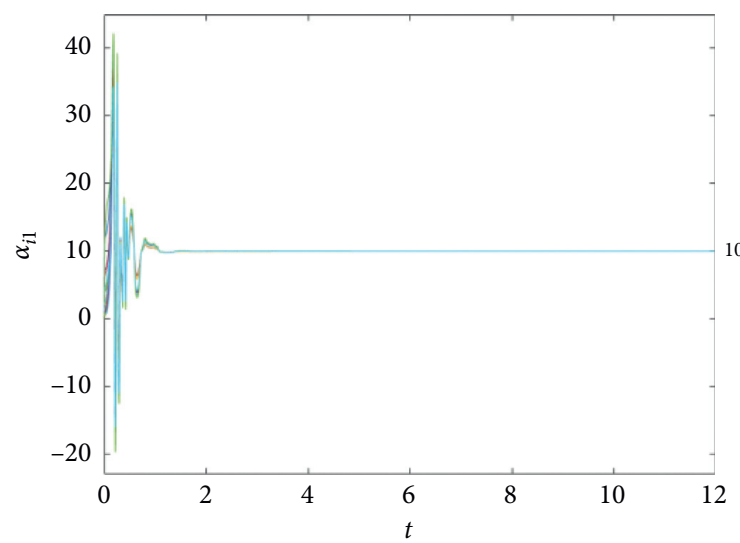

(a)

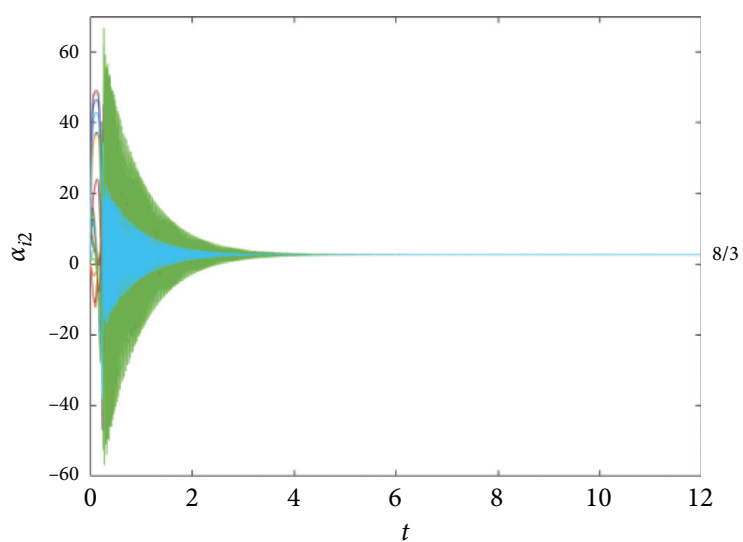

(b)

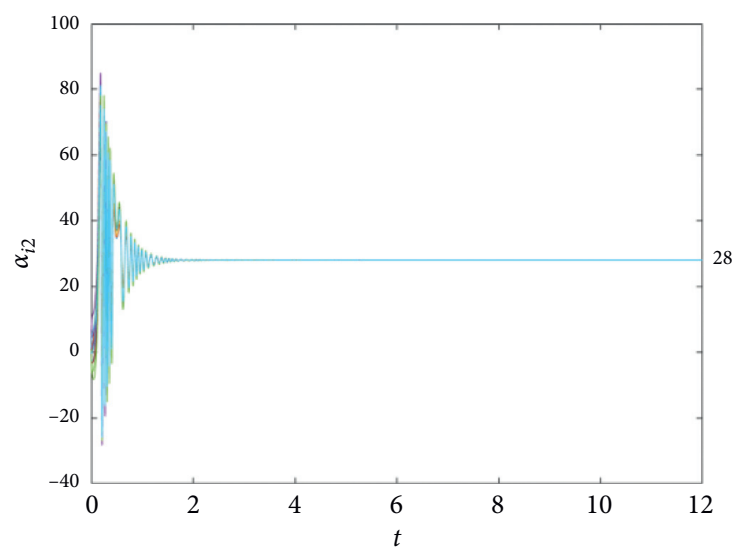

(c)

Figure 3: Identification of the uncertain parameter $\alpha_{i}$.

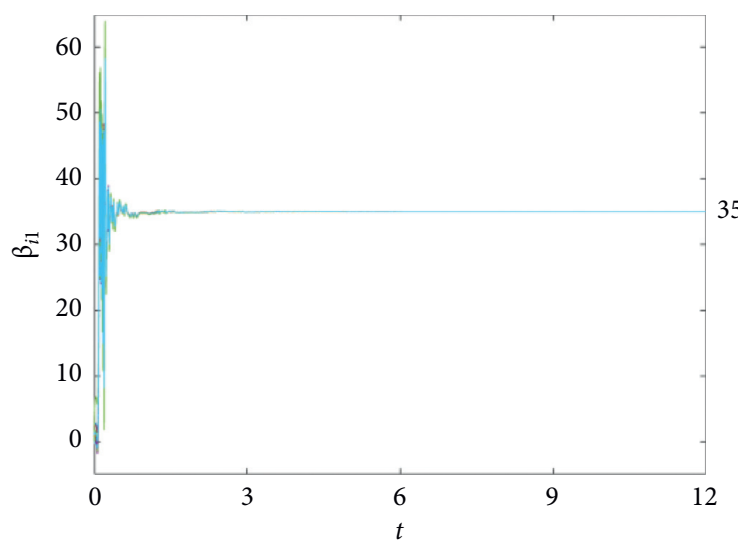

(a)

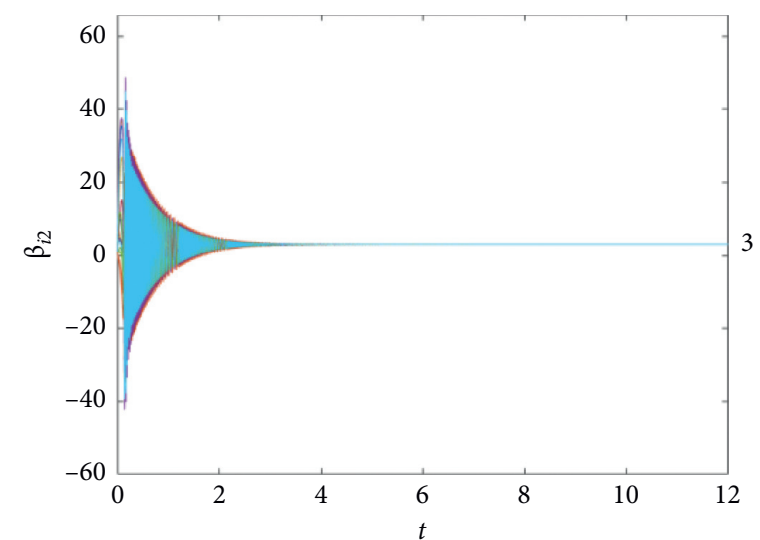

(b)

Figure 4: Continued. 


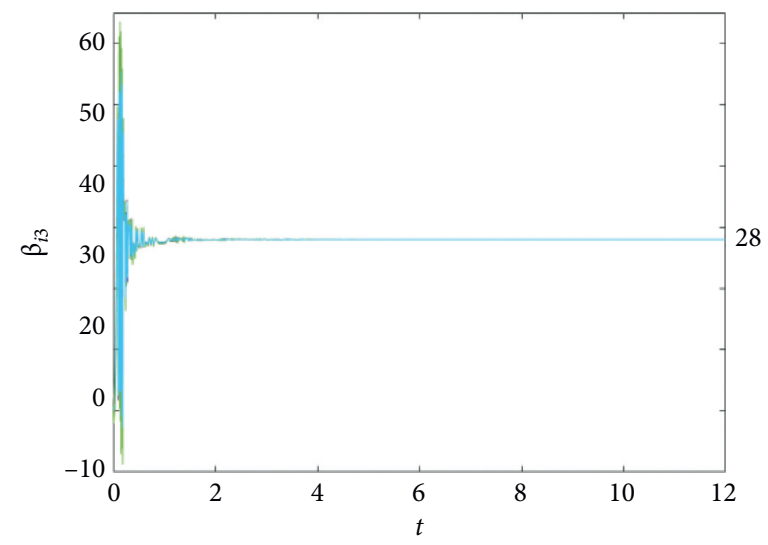

(c)

FIGURE 4: Identification of the uncertain parameter $\beta_{i}$.

\section{Conclusion}

This paper mainly solved the finite-time synchronization and parameter identification problem between two nonlinear coupled Markovian switching complex delayed networks with multiple weights uncertainties. Based on finite-time stability theory and some inequality technologies, an effective controller with update laws is designed to achieve finite-time synchronization of the networks; in the meantime, the unknown parameters are identified in a finite time. Finally, the results of numerical simulation have shown that the synchronization of the networks can be realized at about $t \approx 5$; at the same time, the unknown parameter vector of the networks can also be identified true value $\alpha_{i}=(10,8 / 3,28)^{T}$ and $\beta_{i}=(35,3,28)^{T}$ at about $t \approx 7$. In addition, the feasibility and applicability of the theoretical proof in this paper has been further confirmed.

\section{Data Availability}

The date used to support the findings of this study are included within the article.

\section{Disclosure}

This work was not supported by any fund.

\section{Conflicts of Interest}

The authors declare that they have no conflicts of interest.

\section{Authors' Contributions}

All authors contributed equally to the research and writing of this article. All authors read and approved the final manuscript.

\section{References}

[1] Z. Wang and X. Liu, "Synchronization of interconnected discontinuous neural networks with nonlinear coupling functions," IEEE Access, vol. 7, pp. 25804-25814, 2019.
[2] K. Wu, B. Li, Y. Du, and S. Du, "Synchronization for impulsive hybrid-coupled reaction-diffusion neural networks with time-varying delays," Communications in Nonlinear Science and Numerical Simulation, vol. 82, Article ID 105031, 2020.

[3] D. Wang and L. Huang, "Robust synchronization of discontinuous Cohen-Grossberg neural networks: pinning control approach," Journal of the Franklin Institute, vol. 355, no. 13 , pp. $5866-5892,2018$.

[4] C. Yang, T. Huang, K. Yi et al., "Synchronization for nonlinear complex spatio-temporal networks with multiple time-invariant delays and multiple time-varying delays," Neural Processing Letters, vol. 50, no. 2, pp. 1051-1064, 2019.

[5] J. Liu, H. Wu, and J. Cao, "Event-triggered synchronization in fixed time for semi-Markov switching dynamical complex networks with multiple weights and discontinuous nonlinearity," Communications in Nonlinear Science and Numerical Simulation, vol. 90, 2020.

[6] M. Liu, H. Wu, and W. Zhao, "Event-triggered stochastic synchronization in finite time for delayed semi-Markovian jump neural networks with discontinuous activations," Computational and Applied Mathematics, vol. 39, no. 2, 2020.

[7] W. Zhao and H. Wu, "Fixed-time synchronization of semiMarkovian jumping neural networks with time-varying delays," Advances in Difference Equations, vol. 2018, no. 1, p. 213, 2018.

[8] X. Peng, H. Wu, and J. Cao, "Global nonfragile synchronization in finite time for fractional-order discontinuous neural networks with nonlinear growth activations," IEEE Transactions on Neural Networks and Learning Systems, vol. 30, no. 7, pp. 2123-2137, 2019.

[9] Y. Ma, N. Ma, and L. Chen, "Synchronization criteria for singular complex networks with Markovian jump and timevarying delays via pinning control," Nonlinear Analysis: Hybrid Systems, vol. 29, pp. 85-99, 2018.

[10] K. S. Anand, G. A. Harish Babu, M. Syed Ali, and S. Padmanabhan, "Finite-time synchronization of Markovian jumping complex dynamical networks and hybrid couplings," Chinese Journal of Physics, vol. 62, pp. 304-312, 2019.

[11] Q. Liu, B. Kan, Q. Wang, and Y. Fang, "Cluster synchronization of Markovian switching complex networks with hybrid couplings and stochastic perturbations," Physica A-Statistical Mechanics and its Applications, vol. 526, Article ID 120937, 2019. 
[12] X.-L. An, L. Zhang, and J.-G. Zhang, "Research on urban public traffic network with multi-weights based on single bus transfer junction," Physica A: Statistical Mechanics and its Applications, vol. 436, pp. 748-755, 2015.

[13] C. Yi, J. Feng, J. Wang, C. Xu, Y. Zhao, and Y. Gu, "Pinning synchronization of nonlinear and delayed coupled neural networks with multi-weights via aperiodically intermittent control," Neural Processing Letters, vol. 49, no. 1, pp. 141-157, 2018.

[14] C. Zhang, X. Wang, C. Wang, and Z. Liu, "Synchronization in nonlinear complex networks with multiple time-varying delays via adaptive aperiodically intermittent control," International Journal of Adaptive Control and Signal Processing, vol. 33, no. 1, pp. 39-51, 2019.

[15] Y. Liu, Y. Qin, J. Huang, T. Huang, and X. Yang, "Finite-time synchronization of complex-valued neural networks with multiple time-varying delays and infinite distributed delays," Neural Processing Letters, vol. 50, no. 2, pp. 1773-1787, 2018.

[16] C. Zhang, X. Wang, S. Unar, and Y. Wang, "Finite-time synchronization of a class of nonlinear complex-valued networks with time-varying delays," Physica A-Statistical Mechanics and its Applications, vol. 528, Article ID 120985, 2019.

[17] L. Lü, Q. Wei, H. Jia et al., "Parameter identification and synchronization between uncertain delay networks based on the coupling technology," Physica A: Statistical Mechanics and its Applications, vol. 534, Article ID 120713, 2019.

[18] D. Yao, Q. Lu, C. Wu, and Z. Chen, "Robust finite-time state estimation of uncertain neural networks with Markovian jump parameters," Neurocomputing, vol. 159, pp. 257-262, 2015.

[19] R. Z. Luo and Y. L. Wang, "Finite-time modified projective synchronization between two different chaotic systems with parameter and model uncertainties and external disturbances via sliding control," Indian Journal of Physics, vol. 88, no. 3, pp. 301-309, 2014.

[20] F. Xiao, Q. Gan, and Q. Yuan, "Finite-time cluster synchronization for time-varying delayed complex dynamical networks via hybrid control," Advances in Difference Equations, vol. 2019, no. 1, 2019.

[21] J. Hou, Y. Huang, and E. Yang, "Finite-time anti-synchronization of multi-weighted coupled neural networks with and without coupling delays," Neural Processing Letters, vol. 50, no. 3, pp. 2871-2898, 2019.

[22] J. Yin, S. Khoo, Z. Man, and X. Yu, "Finite-time stability and instability of stochastic nonlinear systems," Automatica, vol. 47, no. 12, pp. 2671-2677, 2011.

[23] J. Q. Lu and J. Cao, "Synchronizati on-based approach for parameters identification in delayed chaotic neural networks," Physica A-Statistical Mechanics and its Applications, vol. 382, pp. 672-682, 2007.

[24] C. Yuan and X. Mao, "Robust stability and controllability of stochastic differential delay equations with Markovian switching," Automatica, vol. 40, no. 3, pp. 343-354, 2004. 